\title{
Demenzstationen stellen keine sinnvolle Spezialisierung dar - Pro
}

\author{
Dementia Wards are not a Useful Specialisation - Pro
}

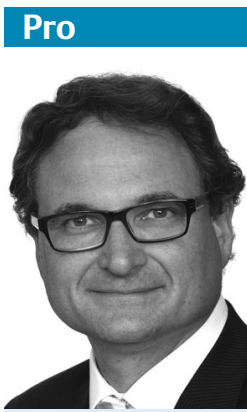

Peter W. Nyhuis
Die stationäre gerontopsychiatrische Versorgung findet in Deutschland ganz überwiegend auf sogenannten „Spezialstationen" statt, zumindest in Kliniken, deren Gesamtbettenanzahl diagnosebezogene Schwerpunktstationen ermöglicht. Auf gerontopsychiatrischen Stationen werden vorwiegend demenzkranke Menschen behandelt, aufgrund deren häufigen Weglaufverhaltens die Stationstüren zumeist geschlossen oder zumindest „fakultativ geschlossen“ sind [1]. Außerdem ist bekannt, dass es gerade auf Demenzstationen zu einer erhöhten Rate von Gewaltvorkommnissen in Form von Fixierungen wegen aggressiven Patientenverhaltens oder durch Anwendung von Bettgittern wegen Sturzgefährdung kommt [2]. Dennoch wird als Hauptargument für die Einrichtung von Demenzstationen regelhaft die angeblich nur in diesem Setting zu gewährleistende ärztliche und pflegerische Qualität ins Feld geführt. Am St. Marien-Hospital Eickel in Herne wird die gleiche Patientenklientel seit vielen Jahren über 6 ausschließlich offen geführte, diagnostisch gemischte Stationen verteilt. Bettgitter gibt es in der Klinik regelhaft nicht und auch kam es in den vergangenen Jahren zu keiner einzigen Fixierung eines demenzkranken Menschen. Die wesentlichen Gründe für diese beeindruckende Reduktion aggressiver Vorkommnisse liegen in der pflegerischen Haltung und Patientenbezogenheit sowie in Maßnahmen zur Bildung eines alltagsnahen, stationären Lebensraums, was nur durch Vermeidung einer gerontopsychiatrischen Schwerpunktbildung auf einer Station zu erreichen ist.

Aus Sicht der Betroffenen macht es am allerwenigsten Sinn, demenzkranke Menschen auf einer Station zu bündeln und so quasi eine künstliche Atmosphäre zu schaffen, die keinerlei Entsprechung im Alltagsleben hat. So ist doch bekannt, dass durch das Ausbleiben alltagsnaher, gesunder Stimuli nicht nur die Resilienzförderung wegfällt, sondern vielmehr Verunselbstständigung, kognitive Verschlechterung und auch eine Zunahme affektiver Begleitsymptome resultieren [3]. Zudem fällt bei räumlicher Bündelung von Demenzkranken gänzlich eine wohlwollende, das Stationsklima erheblich entspannende Fürsorge unter den Patienten weg. Vielmehr muss diese dann ausschließlich von den professionellen Pflegekräften übernommen werden, was aber weder atmosphärisch das Gleiche ist noch regelhaft gelingt. Es überrascht, dass diese negativen Effekte von Demenzstationen von weiten Teilen der Fachwelt völlig ausgeblendet werden, wo doch bei anderen Diagnosegruppen der positive Einfluss der Mitpatienten auf den Genesungsprozess als elementar betrachtet und als ein vermeintliches Hauptargument für Spezialstationen - z.B. DBT-Stationen - angeführt wird.

Durch Verteilung der pflegeintensiven Patienten auf mehrere Stationen lässt sich nicht nur generell ein natürlicheres Milieu unter Vermeidung typischer Krankenhausatmosphäre herstellen, auch die Pflege kann deutlich zugewandter und unerschöpfter erfolgen, wenn sich nur einige Demenzkranke auf einer Station befinden [4]. 1:1-Betreuungen, teilweise im stündlichen Wechsel, sind besser zu leisten und auch für das Personal emotional weniger belastend. Da sich zudem die Pflegekräfte aufgrund der diagnostisch gemischten Stationsbelegung im Gegensatz zu Demenzstationen ständig auf unterschiedlichen - auch unbeeinträchtigten Kommunikationsebenen bewegen müssen, wird insgesamt eine fürsorgliche, patientenzugewandte Haltung des Personals gefördert, z.B. auch durch Vermeidung eines typischen, von Abgrenzung geprägten Kliniksoziolektes [5]. Bei besonders personalintensiven Situationen können kurzfristig Pflegekräfte von anderen Stationen helfen, da die Mitarbeiter der gesamten Klinik ja im Umgang mit gerontopsychiatrischen Patienten geschult und erfahren sind. Schreiende Demenzkranke sind nur selten auf den Stationen zu hören, da ihnen Platz am Tisch des Dienstzimmers angeboten wird, wo sie oft ruhig und deutlich zugänglicher werden. Sedierende Tagesmedikation muss seltener eingesetzt werden, nächtlich unruhige Demenzpatienten sind eher die Ausnahme als die Regel.

Diagnostik und Therapie erfolgen auch bei den auf mehrere Stationen verteilten Demenzkranken leitliniengerecht, die Gruppeneinheiten stationsübergreifend. So ist störungsspezifische Behandlung gewährleistet, zumal gerontopsychiatrisch besonders geschulte Mitarbeiter für diese Patientenklientel in der gesamten Klinik zuständig sind. Beispielsweise sind manche Sozialarbeiter schwerpunktmäßig klinikweit für Aufgaben zur Heimunterbringung, Organisation von Kurzzeitoder häuslicher Pflege, Angehörigenschulung oder Demenznetzwerkarbeit zuständig. Das für Demenzstationen geläufige Argument der erhöhten medizinischen Qualität trifft somit in keiner Weise zu. Aus den angeführten Gründen der Patientenzugewandtheit und der positiven Haltung der Pflege bieten gemischte Stationen für Demenzpatienten auch aus Sicht der Pflegequalität nur Vorteile, wohingegen gerontopsychiatrische Spezialstationen diesbezüglich eher gefährdet sind.

Spätestens seit der UN-Konvention zu den Rechten von Menschen mit Behinderungen unterliegen institutionelle Maßnahmen, die den Umfang von Selbstbestimmung einschränken, einer besonderen Infragestellung. Dies betrifft auch demenzkranke Menschen mit der Fragestellung, ob sie üblicherweise auf einer offenen oder einer geschlossenen Station behandelt werden. Die Verteilung akut kranker Patienten und somit auch pflegeintensiver, weglaufgefährdeter Demenzkranker über alle Stationen ermöglicht eine Klinik 
der konsequent geöffneten Türen [6]. Dahingegen sind die meisten Demenzstationen - mindestens „fakultativ“ - geschlossen. Allein diese Tatsache gibt schon Anlass zum Überdenken, hinzu kommt ein nicht selten entstehender und nur auf der geschlossenen Tür beruhender, ungewollter Mechanismus: Für als nicht geschäftsfähig eingeschätzte Patienten, die ihren freiwilligen Verbleib auf der geschlossenen Station nicht testieren können, wird nicht selten eine gesetzliche Betreuung eingeleitet, wohingegen sich auf einer offenen Station diese Frage bei demselben Patienten nicht stellen würde [7]. Hier begründet sich der Anlass zur Betreuungseinrichtung allein im Ausmaß der hirnorganischen Beeinträchtigung und nicht zusätzlich in der Klinikstruktur.

Die Vermeidung von Demenzstationen in psychiatrischen Kliniken sollte die Regel und nicht die Ausnahme sein. Im Interesse der erkrankten Menschen, ihrer Angehörigen, aber auch der Klinikmitarbeiter gibt es hierfür nur gute Gründe. Diese Einschätzung beruht nicht nur auf einer regionalen Herner Besonderheit, sondern erfolgte so auch schon an anderer Stelle [8]. Der Vorwurf eines Qualitätsverlustes durch Verzicht auf eine Spezialstation ist ungerechtfertigt und sachlich falsch, vielmehr spricht vieles dafür, dass mindestens die Pflegequalität sogar steigt, wenn man eine Kumulation von Demenzkran- ken auf einer Station vermeidet. Betrachtet man zudem die deutlich höheren Fixierungszahlen auf gerontopsychiatrischen gegenüber durchmischten Stationen [9] und vergegenwärtigt sich ihre Verursachung durch deren spezifische Struktur, bedarf es für die Rechtfertigung von Demenzstationen schon durchschlagender Argumente. Aus meiner Sicht gibt es solche nicht.

\section{Literatur}

1 Haglund $K$, van der Meiden E, von Knorring $L$ et al. Psychiatric care behind locked doors. A study regarding the frequency of and the reasons for locked psychiatric wards in Sweden. J Psychiatr Ment Health Nurs 2007; 14: 49-54

2 Sampson EL, White N, Leurent B et al. Behavioural and psychiatric symptoms in people with dementia admitted to the acute hospital: prospective cohort study. Br J Psychiatry 2014; 205: 189-196

3 Watkin L, Blanchard MR, Tookman A et al. Prospective cohort study of adverse events in older people admitted to the acute general hospital: risk factors and the impact of dementia. Int J Geriatr Psychiatry 2012; 27 : $76-82$

4 Oh YS, Lee JE, Lee PH et al. Neuropsychiatric symptoms in Parkinson's disease dementia are associated with increased caregiver burden. J Mov Disord 2015; 8: 26-32

5 Ostaszkiewicz J, Lakhan P, O'Connell B et al. Ongoing challenges responding to behavioural and psychological symptoms of dementia. Int Nurs Rev 2015; Feb 25
6 Lanza ML, Kayne HL, Hicks $C$ et al. Environmental characteristics related to patient assault. Issues Ment Health 1994; 15: 319335

7 Hasle NJ. Involuntary commitment in psychiatry of persons aged 65 and older. Ugeskr Laeger 1996; 158: 2692-2696

8 Gebhardt RP, Radtke M. Vergleich der Stationsatmosphäre zwischen drei Spezialstationen für alkoholkranke, schizophrene und depressive Patienten und einer diagnostisch gemischten Satellitenstation. Psychiat Prax 2003; 30: $192-198$

9 Chou KR, Kaas MJ, Richie MF. Assaultive behavior in geriatric patients. J Gerontol Nurs 1996; 22: 30-38

\section{Korrespondenzadresse}

\section{Dr. Peter W. Nyhuis}

Chefarzt, St. Marien-Hospital Eickel,

Klinik für Psychiatrie, Psychotherapie und

Psychosomatik

Marienstraße 2

44651 Herne

p.nyhuis@marienhospital-eickel.de

Bibliografie

Dol http://dx.doi.org/

10.1055/s-0034-1387616

Psychiat Prax 2015; 42: 178-179

(c) Georg Thieme Verlag KG

Stuttgart · New York

ISSN 0303-4259 